\title{
MARTINGALES OF BANACH-VALUED RANDOM VARIABLES ${ }^{1}$
}

\author{
BY S. D. CHATTERJI
}

Communicated by J. L. Doob, July 18, 1960

1. Let $(\Omega, \Theta, P)$ be a probability space and let $x$ be a Banach space. Let $X(\omega)$ be a Bochner-integrable function on $(\Omega, \beta, P)$ taking values in $X$. Let $\mathcal{F}$ be a Borel-field contained in $B$. We define $E(X \mid \mathcal{F})$, "a conditional expectation of $X(\omega)$ relative to $\mathscr{F}$ " as a Bochnerintegrable function on $(\Omega, \Theta, P)$ (henceforth called random variable or r.v.) such that $E\{X \mid \mathcal{F}\}$ is measurable $\mathcal{F}$ and that

$$
\int_{A} E(X \mid \mathfrak{F}) d P=\int_{A} X d P, \quad A \in \mathcal{F},
$$

where the integrals are Bochner-integrals.

Let $I$ be a subset of the set of all integers and let $X_{i}(\omega), i \in I$ be Bochner-integrable $X$-valued r.v.'s. Let $\mathcal{F}_{i} \subset ß, i \in I$ be a set of Borelfields such that

$$
\mathfrak{F}_{i} \subset \mathfrak{F}_{j} \quad \text { if } i<j .
$$

The triple $\left\{X_{i}, F_{i}, i \in I\right\}$ will be called a martingale if whenever

$$
\begin{gathered}
i<j, i, j \in I, \\
X_{i}=E\left(X_{j} \mid \Im_{i}\right), \text { a.e. }
\end{gathered}
$$

If $Z(\omega)$ is any Bochner-integrable r.v. and if we define

$$
X_{i}=E\left(Z \mid \Im_{i}\right)
$$

then it follows directly from above that $\left\{X_{i}, \mathcal{F}_{i}, i \in I\right\}$ is a martingale.

By $L_{p}(\Omega, \mathcal{F}, P, \mathfrak{X}), 1 \leqq p \leqq \infty$, we shall denote the class of those $X$-valued functions $X(\omega)$ which are strongly-measurable w.r.t. $\mathcal{F}$ and such that $\|X(\omega)\|$ is in $L_{p}$. If we define the "norm" of $X(\omega)$ $\in L_{p}(\Omega, \mathcal{F}, P, \mathscr{X})$, denoted by $[X]_{p}$, to be the $L_{p}$ norm of $\|X(\omega)\|$, then, as is well-known, $L_{p}(\Omega, \mathcal{F}, P, \mathscr{X})$ becomes a Banach space.

A set of complex-valued r.v.'s $X_{t}(\omega), t \in T$ will be said to be "restrictedly uniformly integrable" ${ }^{2}$ if for any $\epsilon>0$ there exists a $\delta>0$ such that

1 Research done under a grant from the National Science Foundation.

${ }^{2}$ I am indebted to Professor Doob for having suggested this terminology in one of his letters to me. I also thank him for pointing out to me that our Theorem 1 had been obtained by Mrs. Moy before, and that Mr. Scalora obtained results somewhat similar to ours in his doctoral dissertation. 


$$
\int_{A}\left|X_{t}(\omega)\right| d P<\epsilon
$$

wherever $P(A)<\delta$. Notice that for general probability measures (e.g. those measures which contain atoms) restrictedly uniform integrability is a weaker condition than ordinary uniform integrability.

2. We shall state our main results, deferring the proofs to a later publication.

THEOREM 1. For any Bochner-integrable r.v. $X$ and Borel-field $F$ $E(X \mid F)$ exists and is unique except for sets of $P$-measure 0.

In passing we state that the usual properties of a Bochner-integral are valid for $E(X \mid \mathcal{F})$ e.g.

$$
\|X\| \leqq E(\|X\| \mid \Im) \text { a.e., etc. }
$$

Theorem 2. Let $\left\{X_{n}, \Im_{n}, n \geqq 1\right\}$ be a $\Upsilon$-valued martingale such that

$$
X_{n}=E\left(Z \mid \mathfrak{F}_{n}\right), \quad n \geqq 1,
$$

where

$$
Z \in L_{p}(\Omega, \bigotimes, P, x), \quad 1 \leqq p<\infty, x \text { arbitrary. }
$$

Then

$$
\lim _{n \rightarrow \infty}\left[X_{n}-X_{\infty}\right]_{p}=0
$$

where $X_{\infty}=E\left(Z \mid F_{\infty}\right)$ and

$$
\mathfrak{F}_{\infty}=\text { Borel-field generated by } \bigcup_{n=1}^{\infty} \mathcal{F}_{n} .
$$

Theorem 3. Let $\left\{X_{n}, \mathcal{F}_{n}, n \leqq-1\right\}$ be a $x$-valued martingale, $x$-arbitrary, and let $X_{-1} \in L_{p}(\Omega, \leftrightarrow, P, x) 1 \leqq p \leqq \infty$. Then

$$
\lim _{n \rightarrow \infty}\left[X_{-\infty}-X_{-n}\right]_{p}=0
$$

where

$$
X_{-\infty}=E\left(X_{-1} \mid F_{-\infty}\right)
$$

and

$$
\mathcal{F}_{-\infty}=\bigcap_{n=1}^{\infty} \mathcal{F}_{-n}
$$


THEOREM 4. Let $\mathscr{X}$ be a reflexive Banach space and let $\left\{X_{n}, \Im_{n}, n \geqq 1\right\}$ be a $\mathrm{X}$-valued martingale such that

$$
X_{n} \in L_{p}(\Omega, \Theta, P, X) \quad n \geqq 1,1<p<\infty
$$

and

$$
\left[X_{n}\right]_{p}<C .
$$

Then there exists $X_{\infty} \in L_{p}(\Omega, \Theta, P, X)$ such that

$$
\lim _{n \rightarrow \infty}\left[X_{n}-X_{\infty}\right]_{p}=0
$$

and

$$
X_{n}=E\left(X_{\infty} \mid F_{n}\right) .
$$

Theorem 5. Let $\left\{X_{n}, F_{n}, n \geqq 1\right\}$ be a $X$-valued martingale where $X$ is reflexive and let $\left\|X_{n}\right\|, n \geqq 1$ be restrictedly uniformly integrable. Then there is a Bochner-integrable r.v. $X_{\infty}$ such that

$$
\lim _{n \rightarrow \infty}\left[X_{n}-X_{\infty}\right]_{1}=0 .
$$

THEOREM 6. Theorem 2 remains valid if we replace (1) by

$$
\lim _{n \rightarrow \infty} X_{n}=X_{\infty} \text { a.e. }
$$

TheOREM 7. Theorem 3 remains valid if we replace (2) by

$$
\lim _{n \rightarrow \infty} X_{-n}=X_{-\infty} \text { a.e. }
$$

THEOREM 8. Theorems 4 and 5 remain valid if we replace (3) and (4) by

$$
\lim _{n \rightarrow \infty} X_{n}=X_{\infty} \text { a.e. }
$$

Theorem 9. Let $\left\{X_{n}, \mathcal{F}_{n}, n \geqq 1\right\}$ be a $\mathfrak{X}$-valued martingale where $\mathfrak{X}$ is reflexive and let

$$
E\left(\sup _{n \geq 0}\left\|X_{n}-X_{n-1}\right\|\right)<+\infty, \quad X_{0} \equiv 0 .
$$

Then

$$
\begin{aligned}
& \lim _{n \rightarrow \infty} X_{n}(\omega) \text { exists whenever } \\
& \omega \in\left\{\omega: \sup _{n}\left\|X_{n}(\omega)\right\|<+\infty\right\} .
\end{aligned}
$$




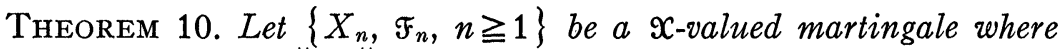
$x$ is reflexive. If $E\left(\left\|X_{n}\right\|\right)<C$, independent of $n$, then there exists a Bochner-integrable r.v. $X_{\infty}(\omega)$ such that $X_{n}(\omega)$ converges weakly to $X_{\infty}(\omega)$ a.e.

TheOREM 11. If $X$ is not reflexive then none of the Theorems $4,5,8$, 9 , and 10 are valid.

For the last theorem we consider $(\Omega, \Theta, P)$ on the open unit interval with Lebesgue measure on Borel subsets, and consider $x$ to be the Banach space of all Lebesgue integrable functions on $(0,1)$. Let $\epsilon_{\lambda}(t) \in \mathcal{X}, 0<\lambda<1$ be as follows:

$$
\begin{array}{rlrl}
\epsilon_{\lambda}(t) & =1, & & 0<t \leqq \lambda, \\
& =0, & \lambda<t<1 .
\end{array}
$$

Let $\Im_{n}$ be the Borel-field generated by intervals

$$
\left(\frac{m}{2^{n}}, \frac{m+1}{2^{n}}\right),
$$

$0 \leqq m \leqq 2^{n}-1, n \geqq 1$. Define

$$
\begin{aligned}
X_{n}(\omega) & =2^{n}\left\{\epsilon_{(m+1) / 2^{n}}-\epsilon_{m / 2^{n}}\right\}, & & \omega \in\left(\frac{m}{2^{n}}, \frac{m+1}{2^{n}}\right), \\
& =0, & & \text { elsewhere. }
\end{aligned}
$$

Then

$$
\left\{X_{n}, \Im_{n}, n \geqq 1\right\}
$$

is a martingale and

$$
\begin{aligned}
& \left\|X_{n}(\omega)\right\| \equiv 1 \text { a.e. } \\
& E\left(\left\|X_{n}(\omega)\right\| p\right)=1, \quad n \geqq 1, \\
& E\left(\sup _{n \geqq 0}\left\|X_{n}(\omega)-X_{n-1}(\omega)\right\|\right)=1, \quad X_{0} \equiv 0 .
\end{aligned}
$$

But if $\omega \neq p / 2^{q}$ then $X_{n}(\omega)$ does not go to any limit either weakly or strongly. Actually no subsequence $X_{n_{k}}(\omega)$ converges weakly or strongly if $\omega \neq p / 2^{q}$. Hence $X_{n}(\omega)$ does not converge in $L_{1}(\Omega, \Theta, P, x)$ mean either.

Michigan State University 